Methods Cross-sectional study of patients with SLE. Demographics, coronary risk, disease activity and inflammatory markers were studied. The diagnosis of MS was established with the NHLBI/AHA criteria. Statistical analysis was performed using SPSS 20.0 software and a $\mathrm{P}$ value $<0.05$ was considered significant.

Results 126 patients with SLE, 107 women (84\%) and 19 men (15\%), age $41 \pm 13$ years old and disease duration $9 \pm 7$ years. The prevalence of MS was 33.3\%. No association was found with age, education level, smoking or steroid use in patients with MS. In multivariate analysis only elevated erythrocyte sedimentation rate (ESR) had a statistical significance $(p=0.012)$. Positive association was found between higher values of ESR and hypertriglyceridemia $(p=0.0002)$, body mass index $(p=0.0043)$ and lower levels of HDL and C3 $(\mathrm{p}=0.0152)$.

Conclusions The prevalence of MS in our population (33\%) was higher than reported in the SLICC registry (15\%). The association of metabolic and inflammatory characteristics increases cardiovascular risk by a proinflamatory state. The results suggest the need for early diagnosis and treatment of MS to reduce cardiovascular comorbidity in patients with SLE.

\section{VITAMIN D DEFICIENCY AS NOVEL RISK FACTOR OF ACCELERATED ATHEROSCLEROSIS CARDIOASCULAR DISEASES IN PATIENTS WITH SYSTEMIC LUPUS ERYTHEMATOSUS}

${ }^{1}$ RA Nugraha, ${ }^{1} F F$ Alkaff, ${ }^{1} \mathrm{M}$ Jonatan, ${ }^{1}$ RB Wicaksono, ${ }^{1} \mathrm{TN}$ Octora, ${ }^{2} \mathrm{R}$ Yudiwati, ${ }^{3} \mathrm{~A}$ Awalia. ${ }^{1}$ Faculty of Medicine Universitas Airlangga, Medicine, Surabaya, Indonesia; ${ }^{2}$ Faculty of Medicine Universitas Airlangga, Biomedics and Biomolecular Medicine, Surabaya, Indonesia; ${ }^{3}$ Faculty of Medicine Universitas Airlangga, Rheumatology, Surabaya, Indonesia

\subsection{6/lupus-2017-000215.188}

Background and aims Vitamin D deficiency is common in women living near to equatorial line. Deficiency of vitamin D could lead to more severe manifestation of autoimmune disease, it may accelerate complication in atherosclerosis cardiovascular disease.
Objective We aimed to evaluate the impact of vitamin D deficiency in the severity of SLE, especially in the appearance of ASCVD in SLE patients.

Methods Design: Systematic review and meta-analysis.

Data Sources: Electronic databases (CENTRAL; Medline; Springerlink; Cochrane Database) were searched up to May 2015.

Review methods: Longitudinal study that compared level of serum vitamin D in SLE patients with and without ASCVD manifestation were included. Study selection, data extraction and risk of bias assessment (Cochrane risk of bias tool) were performed by five reviewers.

Results Total of 16 trials (1723 participants) were included. Meta-analysis of 8 trials (781 participants) found that serum vitamin D (25-hydroxyvitamin D) levels in severe SLE patients with ASCVD manifestation were significantly lower compared to non-ASCVD SLE patients during remission (pooled RR $0.64 ; 95 \% \mathrm{CI}=0.34-0.77 ; \mathrm{p}=0.005)$. No statistically significant difference in serum vitamin $\mathrm{D}$ were observed in metaanalysis of other 8 trials (pooled RR 0.96; 95\% CI=0.54-1.7; $\mathrm{p}=0.12$ ). Moreover, there is a significant inverse correlation between serum vitamin D levels and Systemic Lupus Erythematosus Disease Activity Index $2000(r=-0.373, \mathrm{p}=0.016)$.

Conclusions Results demonstrates that vitamin D deficiency could be a novel risk factor for accelerating endothelial dysfunction and ASCVD in SLE patients. Vitamin D supplementation might modulate an immunosuppressive effects, endothelial repair mechanisms, and endothelial function in SLE patients with significant ASCVD risk.

\section{IMPACT OF DISEASE ACTIVITY ON ORGAN DAMAGE RISK OVER TIME IN SYSTEMIC LUPUS ERYTHEMATOSUS (SLE) -THE HOPKINS LUPUS COHORT}

${ }^{1}$ RT Burge, ${ }^{1} Z$ Xiang, ${ }^{1} \mathrm{~J}$ Paik, ${ }^{2} Y$ Wang, ${ }^{3} \mathrm{~L}$ Magder, ${ }^{4} \mathrm{M}$ Petri ${ }^{*}$. 'Eli Lilly and Company, GPORWE, Indianapolis, USA; ${ }^{2}$ Eli Lilly and Company, GSS, Indianapolis, USA; ${ }^{3}$ University of Maryland School of Medicine, Epidemiology and Public Health, Baltimore MD, USA; ${ }^{4}$ The John Hopkins University School of Medicine, Rheumatology, Baltimore- MD, USA

10.1136/lupus-2017-000215.189

Abstract 189 Table 1 Time-dependent cox proportional hazard models: effect of disease activity on the risk of new organ damage.

\begin{tabular}{|c|c|c|c|c|c|c|}
\hline \multirow[b]{2}{*}{ Variable } & \multicolumn{2}{|c|}{ Model 1} & \multicolumn{2}{|c|}{ Model 2} & \multicolumn{2}{|c|}{ Model 3} \\
\hline & HR $(95 \%$ CI $)$ & P-value & HR $(95 \%$ CI) & P-value & HR (95\% CI) & P-value \\
\hline Age at cohort entry & $1.03(1.03-1.04)$ & $<0.001$ & $1.03(1.03-1.04)$ & $<0.001$ & $1.03(1.03-1.04)$ & $<0.001$ \\
\hline SDI score at cohort entry & $1.06(1.02-1.11)$ & 0.002 & $1.07(1.03-1.11)$ & $<0.001$ & $1.07(1.03-1.11)$ & $<0.001$ \\
\hline Immunosuppressant use during follow-up & $1.23(1.05-1.43)$ & 0.012 & $1.15(0.99-1.35)$ & 0.076 & $1.15(0.98-1.35)$ & 0.079 \\
\hline $\begin{array}{l}\text { Average corticosteroid use ( } \geqslant 7.5 \mathrm{mg} \text { vs. } \\
<7.5 \mathrm{mg})\end{array}$ & $1.74(1.49 \cdot 2.04)$ & $<0.001$ & $1.61(1.37-1.89)$ & $<0.001$ & $1.61(1.37 .1 .89)$ & $<0.001$ \\
\hline $\begin{array}{l}\text { SELENA-SLEDAI score during follow- } \\
\text { up }(\geqslant 6 \text { vs. }<6)\end{array}$ & $1.40(1.17-1.67)$ & 0.012 & $0.82(0.65-1.03)$ & 0.088 & NA & \\
\hline $\begin{array}{l}\text { Proportion of elinic visits with SLEDAI } \\
\text { score } \geqslant 6 \text { during follow-up }\end{array}$ & NA & & $3.82(2.71-5.39)$ & $<0.001$ & $3.14(2.42-4.08)$ & $<0.001$ \\
\hline
\end{tabular}

